\title{
Pertinence de la répartition géographique des professionnels des soins oculovisuels en Alberta : les examens de réfraction réalisés par les opticiens sont-ils nécessaires?
}

\author{
Alyssa Anderson, B.Sc. \\ Gordon Hensel, OD, FAAO \\ Alberta College \\ of Optometrists
}

\begin{abstract}
Résumé
Les examens de réfraction comptent parmi les nombreuses procédures diagnostiques effectuées par les optométristes et les ophtalmologistes en vue de prescrire des lunettes. En Alberta, les optométristes et les ophtalmologistes sont autorisés à effectuer des examens de réfraction et à prescrire des lunettes en fonction des résultats. Les opticiens sont aussi autorisés à effectuer des examens de réfraction sans toutefois avoir le droit de prescrire de lunettes. Cette étude vise à déterminer si la population a besoin que les opticiens soient autorisés à effectuer des examens de réfraction et à prescrire des lunettes en solo compte tenu de la répartition géographique actuelle des optométristes et des ophtalmologistes en Alberta. Pour répondre à cette question, les ratios des optométristes et des ophtalmologistes par rapport à la population pour chacune des cinq régions sanitaires de l'Alberta ont été calculés et comparés aux ratios internationaux. Nos résultats montrent que les cinq régions sanitaires de l'Alberta ont des ratios optométriste-population qui atteignent ou dépassent l'indice de référence international de 1 pour 10000 personnes permettant de répondre aux besoins de la population et que trois des cinq régions sanitaires ont des ratios ophtalmologiste-population équivalents ou supérieurs à l'indice de référence international de 3 pour 100000 personnes qui permet de répondre aux besoins de la population. Ces données indiquent que la répartition des optométristes est adéquate dans la province mais que celle des ophtalmologistes est inadéquate. Compte tenu de la disponibilité actuelle des optométristes et des ophtalmologistes en Alberta, il n'est pas nécessaire que les opticiens soient autorisés à effectuer des examens de réfraction et à prescrire des lunettes en solo.
\end{abstract}

MOTS CLÉS :

Réfraction, optométriste, opticien, ophtalmologiste, indice de référence international, Alberta, besoins de la population

\section{INTRODUCTION}

En Alberta, les optométristes et les ophtalmologistes peuvent effectuer des examens complets de la vue et prescrire des lunettes. Un examen complet de la vue comprend une évaluation des antécédents de santé du patient, de la santé oculaire et systémique et de la fonction visuelle, notamment de la réfraction, de l'acuité, du champ visuel et de la vision binoculaire. Une ordonnance digne de confiance pour des lunettes ne peut être livrée qu'en ayant une compréhension globale de la santé et de la fonction visuelle d'un patient. En Alberta, les opticiens sont autorisés à effectuer des examens de réfraction et, avec l'approbation d'un prescripteur autorisé (optométriste ou ophtalmologiste), à distribuer des lunettes en fonction de l'ordonnance reçue de celui-ci.

Dans cette étude, nous entendons déterminer si la population a besoin que les opticiens soient autorisés à effectuer des examens de réfraction et à prescrire des lunettes en solo. Une répartition adéquate des optométristes et des 
ophtalmologistes est importante pour s'assurer que les Albertains, où qu'ils soient établis, aient facilement accès à un professionnel en mesure d'effectuer un examen complet de la vue et d'émettre une ordonnance pour des lunettes.

Cette étude ne permettra pas de déterminer si les opticiens possèdent les connaissances, les habiletés et les compétences requises pour effectuer un examen de réfraction et prescrire des lunettes en fonction de cet examen. De plus, cette étude ne permet pas de déterminer si la population comprend la différence entre un examen de réfraction (test de la vue) et un examen complet de la vue. Bien qu'il s'agisse de deux considérations importantes, seule la question de savoir s'il y a actuellement un nombre suffisant de professionnels de la santé autorisés à fournir des soins en cas d'erreur de réfraction dans la province est étudiée ici.

\section{MÉTHODES}

Pour établir la répartition géographique des professionnels des soins oculovisuels en Alberta, nous avons divisé la province en cinq régions sanitaires. Les régions sanitaires sont définies par les services de santé de l'Alberta (Alberta Health Services) et comprennent la région Nord, la région d'Edmonton, la région Centre, la région de Calgary et la région Sud (figure 1) $)^{1}$.

Figure 1 : Carte de la province de l'Alberta montrant les cinq régions sanitaires définies par les services de santé de l'Alberta. Figure générée à l'aide de l'application de cartographie GeoDiscover Alberta.

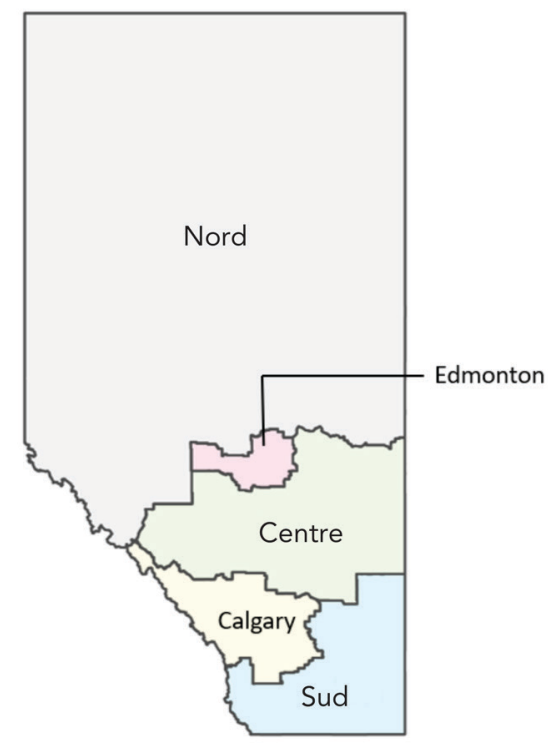

Le nombre et l'emplacement des optométristes, des ophtalmologistes et des opticiens en exercice en Alberta ont été déterminés à l'aide des données de l'ordre des optométristes de l'Alberta (Alberta College of Optometrists), de l'ordre des médecins et chirurgiens de l'Alberta (College of Physicians and Surgeons of Alberta) et de l'ordre et de l'association des opticiens de l'Alberta (Alberta College and Association of Opticians) ${ }^{4}$. D'après les données démographiques de 2018 pour chacune des cinq régions sanitaires, les ratios entre optométristes et ophtalmologistes pour la population de chaque région ont été calculés et comparés aux indices de référence internationaux définis précédemment. L'indice de référence international pour les optométristes est de 1 pour 10000 personnes comme dans le reste du Canada, aux États-Unis et en Australie ${ }^{5,6}$. L'indice de référence international pour les ophtalmologistes est estimé à 3 pour 100000 personnes 7 . Les besoins de la population en matière d'examens de réfraction réalisés par des opticiens sont fondés sur la question de savoir si les ratios des optométristes et des ophtalmologistes par rapport à la population dans chaque région sanitaire respectent ces indices de référence internationaux.

\section{RÉSULTATS}

La figure 2 montre les ratios optométriste-population dans chacune des cinq régions sanitaires de l'Alberta (Nord, Edmonton, Centre, Calgary et Sud) et compare ces ratios à l'indice de référence international précédemment défini de 1 pour 10000 personnes. 
Avec 2,2 optométristes pour 10000 habitants, la région de Calgary affiche le ratio optométriste-population le plus élevé (figure 2). La région d'Edmonton suit de près avec 2,1 optométristes pour 10000 habitants.

Figure 2 : Nombre d'optométristes pour 10000 personnes dans chacune des cinq régions sanitaires de l'Alberta comparativement à l'indice de référence international de 1 pour 10000 personnes. Les données concernant les optométristes sont celles de 2019. Les données sur la population sont tirées du rapport annuel 2018-2019 des services de santé de l'Alberta.

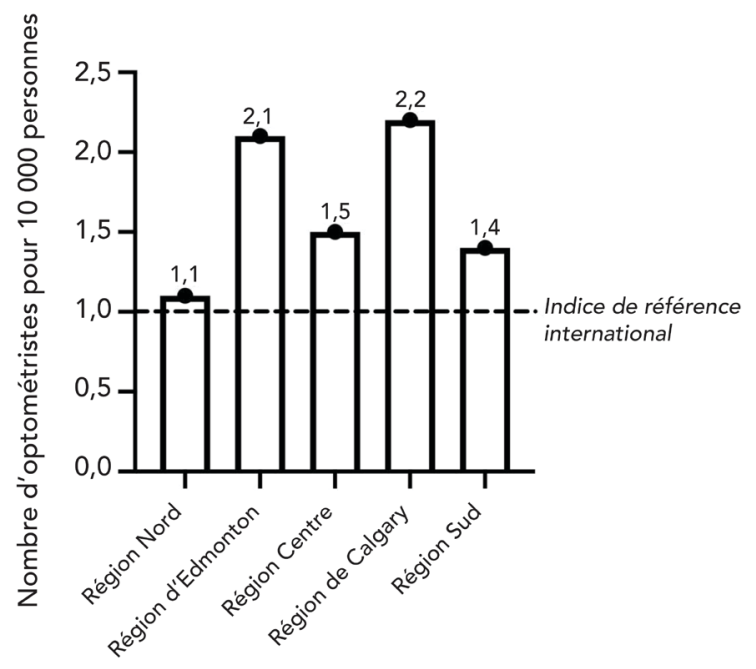

La figure 3 montre les ratios ophtalmologiste-population dans chacune des cinq régions sanitaires de l'Alberta et compare ces ratios à l'indice de référence international précédemment défini de 3 pour 100000 personnes.

Figure 3 : Nombre d'ophtalmologistes pour 100000 personnes dans chacune des cinq régions sanitaires de l'Alberta comparativement à l'indice de référence international de 3 pour 100000 personnes. Le nombre d'ophtalmologistes est celui de 2019. Les données sur la population sont tirées du rapport annuel 2018-2019 des services de santé de l'Alberta.

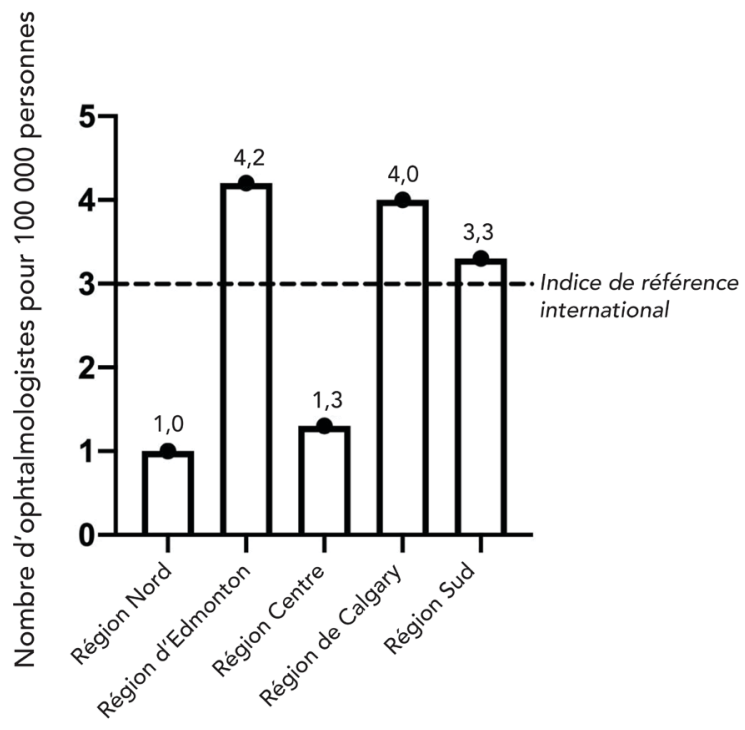


Avec 4,2 ophtalmologistes pour 100000 habitants, la région d'Edmonton affiche le ratio ophtalmologiste-population le plus élevé (figure 3). La région de Calgary suit de près avec 4 ophtalmologistes pour 100000 habitants. En particulier, la région du Nord présente les plus faibles ratios optométriste-population et ophtalmologiste-population des cinq régions [1,1 et 1,0, respectivement] (figures 2 et 3 ).

\section{DISCUSSION}

Lorsque nous avons calculé les ratios des optométristes et des ophtalmologistes par rapport à la population, les données démographiques de 2018 ont été utilisées pour chacune des régions sanitaires de l'Alberta et les données de 2019 ont été utilisées pour déterminer l'emplacement des optométristes et des ophtalmologistes qui exercent actuellement dans la province. Ainsi, les ratios des optométristes et des ophtalmologistes par rapport à la population ont été calculés à partir de données d'années différentes. Cette limite n'a pu être évitée en raison de l'insuffisance des données publiques sur la population de 2019 pour les régions sanitaires de l'Alberta. Bien que cette limite ait pu causer de légères inexactitudes dans le calcul des ratios des optométristes et des ophtalmologistes par rapport à la population, nous avons jugé qu'elle n'a pas d'incidence importante sur nos conclusions générales.

La figure 2 montre que les ratios optométriste-population des cinq régions sanitaires de l'Alberta atteignent ou dépassent l'indice de référence international de 1 pour 10 000. La figure 3 montre que seulement trois des cinq régions sanitaires ont des ratios ophtalmologiste-population qui atteignent ou dépassent l'indice de référence international de 3 pour 100 000. Plus précisément, les ratios ophtalmologiste-population des régions Nord et Centre sont inférieurs à l'indice de référence international [1,1 et 1,3 respectivement] (figure 3). Une pénurie d'ophtalmologistes dans une région sanitaire particulière peut avoir de graves répercussions sur la capacité de la population d'obtenir des soins oculovisuels d'urgence, exposant ainsi la population à un risque accru de perte de vision évitable. De plus, la répartition inadéquate des ophtalmologistes, combinée à la répartition adéquate des optométristes, donne à penser qu'il serait utile d'élargir le champ d'exercice des optométristes pour qu'ils puissent effectuer certaines interventions chirurgicales ou fournir des traitements actuellement réservés aux ophtalmologistes. Toutefois, cette perspective exige une recherche approfondie et dépasse la portée de notre étude. Fait à noter, la constatation selon laquelle le ratio ophtalmologiste-population est inférieur à l'indice de référence international dans deux régions sanitaires ne signifie pas nécessairement que la population a besoin que les opticiens soient autorisés à effectuer des examens de réfraction et à prescrire des lunettes en solo. En soi, le ratio optométriste-population de 1 pour 10000 est suffisant pour répondre aux besoins de la population en matière d'accès aux soins en cas d'erreur de réfraction. Cette lacune dans le nombre d'ophtalmologistes indique qu'il pourrait y avoir un accès limité aux services de soins oculovisuels de deuxième ligne et aux interventions chirurgicales dans certaines régions sanitaires de l'Alberta plutôt qu'une lacune dans l'offre de soins en cas d'erreur de réfraction. Compte tenu de la répartition géographique seulement, le fait que les cinq régions sanitaires aient des ratios optométriste-population supérieurs à l'indice de référence international étaye la prémisse soutenant qu'il n'est pas urgent, pour la population, que les opticiens effectuent des examens de réfraction et prescrivent des lunettes en solo.

Certes, il y a d'autres facteurs que la répartition géographique dont il faut tenir compte lorsqu'on détermine si la population a besoin que les opticiens effectuent de tels examens et livrent des ordonnances. Dans le cadre d'une étude distincte (non publiée à l'heure actuelle), nous avons effectué une recension des lois régissant les examens de réfraction par les opticiens dans 34 administrations. Nous avons constaté que le modèle d'excellence entourant les examens de réfraction réalisés par des opticiens consiste à les interdire complètement. De plus, une autre étude ${ }^{8}$ nous a permis de constater que les opticiens de l'Alberta ne possèdent pas la formation ni les compétences et les connaissances adéquates pour effectuer des examens de réfraction et prescrire de façon sécuritaire et compétente. Bien que cela dépasse la portée de notre étude actuelle, les conclusions de ces deux études semblent appuyer la conclusion selon laquelle la population n'a pas besoin que les opticiens effectuent des examens de réfraction et prescrivent des lunettes en Alberta.

Si la population de l'Alberta augmente plus rapidement que le nombre d'optométristes et d'ophtalmologistes exerçants, la population pourrait avoir besoin que les opticiens soient autorisés à effectuer des examens de réfraction et à prescrire des lunettes en solo. Toutefois, en 2019, les optométristes et les ophtalmologistes étaient répartis de façon adéquate dans la province pour s'assurer que les Albertains aient facilement accès à un professionnel des soins oculovisuels qui peut effectuer un examen complet de la vue et prescrire des lunettes. 


\section{CONCLUSION}

La répartition géographique des optométristes et des ophtalmologistes dans les cinq régions sanitaires de l'Alberta est adéquate pour garantir que tous les Albertains ont un accès approprié à un professionnel des soins oculovisuels qui peut livrer une ordonnance pour des lunettes. Ces résultats indiquent qu'il n'est pas urgent, pour la population, que les opticiens soient autorisés à prescrire des lunettes et à effectuer des examens de réfraction en solo.

\section{CORRESPONDENCE:}

D ${ }^{\mathrm{r}}$ Gordon Hensel, OD. Courriel : registrar@collegeofoptometrists.ab.ca

\section{RÉFÉRENCES}

1. Alberta Health Services. Alberta Health Services Annual Report 2018-2019. 2019. https://www.albertahealthservices.ca/about/ Page2220.aspx

2. Alberta College of Optometrists. Find an Optometrist. https://aco. alinityapp.com/WebClient/RegistrantDirectory.aspx. Accessed August 4, 2019.

3. College of Physicians and Surgeons of Alberta. Find a Physician. https://search.cpsa.ca/PhysicianSearch. Accessed August 2, 2019.

4. Alberta College and Association of Opticians. Member Roster. https://www.opticianry.ca/MemberRoster/MemberRoster. aspx?OrgID=ACAO. Accessed August 2, 2019.

5. Aldebasi YH, Ahmed MI, Monaco WA. Are optometrists necessary in primary health care centres in Saudi Arabia? African Vis Eye Heal 2018;77(1):1-10. doi:10.4102/aveh.v77i1.402
6. Holden B, Resnikoff S. The role of optometry in vision 2020. Community Eye Health 2002;15(43):33-36. http://www.ncbi.nlm.nih.gov/ pubmed/17491876.

7. Al Ali A, Hallingham S, Buys YM. Workforce supply of eye care providers in Canada: Optometrists, ophthalmologists, and subspecialty ophthalmologists. Can J Ophthalmol 2015;50(6):422-428. doi:10.1016/j.jcjo.2015.09.001

8. Anderson A, Hensel G. Assessing the skills of Alberta's refracting opticians: Can opticians safely and independently refract and prescribe optical appliances? Can J Optom 2021 83(1):13-23. https:// doi.org/10.15353/cjo.v83il.1711

Annexe A : Données démographiques de 2018 pour les régions sanitaires de l’Alberta

\begin{tabular}{|c|c|}
\hline $\begin{array}{c}\text { Région sanitaire des services } \\
\text { de santé de l'Alberta }\end{array}$ & Population \\
\hline Nord & 482635 \\
\hline Edmonton & 1404498 \\
\hline Centre & 479435 \\
\hline Calgary & 1669272 \\
\hline Sud & 306577 \\
\hline Total & 4342417 \\
\hline
\end{tabular}

AHS, Services de santé de l’Alberta 
Annexe B : Données sur l'emplacement des optométristes, des ophtalmologistes et des opticiens en 2019

\begin{tabular}{|c|c|c|c|c|}
\hline Ville & Optométristes & Ophtalmologistes & Opticiens & Région sanitaire \\
\hline Airdrie & 12 & 1 & 14 & Calgary \\
\hline Athabasca & 1 & 0 & 0 & Nord \\
\hline Balzac & 0 & 0 & 1 & Calgary \\
\hline Banff & 0 & 0 & 1 & Calgary \\
\hline Barrhead & 1 & 0 & 1 & Nord \\
\hline Beaumont & 1 & 0 & 3 & Edmonton \\
\hline Beaverlodge & 1 & 0 & 0 & Nord \\
\hline Black Diamond & 1 & 0 & 0 & Calgary \\
\hline Bonnyville & 1 & 0 & 1 & Nord \\
\hline Bragg Creek & 3 & 0 & 2 & Calgary \\
\hline Brooks & 2 & 0 & 2 & Sud \\
\hline Calgary & 324 & 64 & 333 & Calgary \\
\hline Calmar & 1 & 0 & 0 & Edmonton \\
\hline Camrose & 4 & 0 & 0 & Centre \\
\hline Canmore & 6 & 0 & 3 & Calgary \\
\hline Cardston & 2 & 0 & 0 & Sud \\
\hline Chestermere & 5 & 0 & 2 & Calgary \\
\hline Cochrane & 4 & 0 & 8 & Calgary \\
\hline Cold Lake & 3 & 0 & 5 & Nord \\
\hline Comté de Grande Prairie & 3 & 0 & 0 & Nord \\
\hline Devon & 1 & 0 & 0 & Edmonton \\
\hline DeWinton & 1 & 0 & 0 & Calgary \\
\hline Drumheller & 5 & 0 & 4 & Centre \\
\hline Duncan & 0 & 1 & 0 & Nord \\
\hline Edmonton & 223 & 57 & 340 & Edmonton \\
\hline Edson & 3 & 0 & 6 & Nord \\
\hline Fort MacLeod & 1 & 0 & 0 & Sud \\
\hline Fort McMurray & 5 & 0 & 2 & Nord \\
\hline Fort Saskatchewan & 7 & 0 & 9 & Edmonton \\
\hline Gibbons & 1 & 0 & 1 & Edmonton \\
\hline Grande Prairie & 16 & 3 & 25 & Nord \\
\hline High Level & 1 & 0 & 0 & Nord \\
\hline High Prairie & 0 & 0 & 1 & Nord \\
\hline High River & 3 & 1 & 3 & Calgary \\
\hline Hinton & 4 & 0 & 0 & Nord \\
\hline Jasper & 1 & 0 & 0 & Nord \\
\hline Lac La Biche & 1 & 0 & 0 & Nord \\
\hline Lacombe & 3 & 0 & 1 & Centre \\
\hline
\end{tabular}




\begin{tabular}{|c|c|c|c|c|}
\hline Lamont & 0 & 1 & 0 & Centre \\
\hline Leduc & 5 & 0 & 7 & Edmonton \\
\hline Lethbridge & 22 & 6 & 44 & Sud \\
\hline Lloydminister & 10 & 0 & 16 & Centre \\
\hline Maskwacis & 0 & 0 & 2 & Centre \\
\hline Medicine Hat & 11 & 4 & 24 & Sud \\
\hline Morinville & 1 & 0 & 3 & Edmonton \\
\hline Nanton & 0 & 0 & 1 & Calgary \\
\hline Okotoks & 5 & 0 & 19 & Calgary \\
\hline Olds & 3 & 0 & 5 & Centre \\
\hline Oyen & 1 & 0 & 2 & Sud \\
\hline Peace River & 2 & 0 & 0 & Nord \\
\hline Pincher Creek & 1 & 0 & 0 & Sud \\
\hline Ponoka & 2 & 0 & 1 & Centre \\
\hline Red Deer & 25 & 5 & 41 & Centre \\
\hline Comté de Red Deer & 4 & 0 & 0 & Centre \\
\hline Redcliff & 1 & 0 & 0 & Sud \\
\hline Rimbey & 1 & 0 & 0 & Centre \\
\hline Rocky Mountain House & 0 & 0 & 1 & Centre \\
\hline Rocky View & 2 & 0 & 17 & Calgary \\
\hline Sherwood Park & 24 & 1 & 46 & Edmonton \\
\hline Slave Lake & 1 & 0 & 0 & Nord \\
\hline Smoky Lake & 1 & 0 & 0 & Nord \\
\hline Spruce Grove & 7 & 0 & 15 & Edmonton \\
\hline Saint Paul & 4 & 1 & 4 & Nord \\
\hline St. Albert & 15 & 1 & 28 & Edmonton \\
\hline Stettler & 2 & 0 & 2 & Centre \\
\hline Stony Plain & 2 & 0 & 2 & Edmonton \\
\hline Strathmore & 1 & 0 & 1 & Calgary \\
\hline Comté de Sturgeon & 1 & 0 & 0 & Edmonton \\
\hline Sylvan Lake & 0 & 0 & 2 & Centre \\
\hline Taber & 2 & 0 & 5 & Sud \\
\hline Three Hills & 1 & 0 & 0 & Centre \\
\hline Tofield & 1 & 0 & 2 & Centre \\
\hline Vegreville & 2 & 0 & 2 & Centre \\
\hline Vermillion & 1 & 0 & 0 & Centre \\
\hline Wainwright & 5 & 0 & 3 & Centre \\
\hline Westlock & 1 & 0 & 0 & Nord \\
\hline Wetaskiwin & 5 & 0 & 2 & Centre \\
\hline Whitecourt & 2 & 0 & 0 & Nord \\
\hline TOTAL & 825 & 146 & 1065 & \\
\hline
\end{tabular}

